\title{
Nutrição e produtividade da nogueira-macadâmia em função de doses de nitrogênio
}

\author{
Marcos José Perdoná(1), Adriana Novais Martins(2), Eduardo Suguino(3) \\ e Rogério Peres Soratto(4)
}

\begin{abstract}
(1)Agência Paulista de Tecnologia dos Agronegócios (Apta), Regional Centro-Oeste, Avenida Rodrigues Alves, no 40, CEP 17030-000 Bauru, SP. E-mail: marcos.perdona@yahoo.com.br (2)Apta, Regional Centro-Oeste, Rua Andrade Neves, № 81, CEP 17515-400 Marília, SP. E-mail: adrianamartins@apta.sp.gov.br ${ }^{(3)}$ Apta, Regional Centro-Leste, Avenida Bandeirantes, no 2.419, CEP 14030-670 Ribeirão Preto, SP. E-mail: esuguino@apta.sp.gov.br (4)Universidade Estadual Paulista, Faculdade de Ciências Agronômicas, Departamento de Produção e Melhoramento Vegetal, Campus de Botucatu, Caixa Postal 237, CEP 18603-970 Botucatu, SP. E-mail: soratto@fca.unesp.br
\end{abstract}

Resumo - O objetivo deste trabalho foi avaliar a influência de doses de nitrogênio na nutrição mineral e na produtividade da nogueira-macadâmia (Macadamia integrifolia). O experimento foi conduzido durante três anos agrícolas, em Jaboticabal, SP. Utilizou-se o delineamento experimental de blocos ao acaso, com quatro repetições. Foram avaliadas cinco doses de $\mathrm{N}\left(0,50,100,150\right.$ e $200 \mathrm{~kg} \mathrm{ha}^{-1}$ por ano), aplicadas na forma de ureia. $\mathrm{O}$ aumento nas doses de $\mathrm{N}$ aumentou o teor do nutriente nas folhas e a produtividade de nozes e amêndoas. Os teores de $\mathrm{Ca}, \mathrm{Mg}$ e $\mathrm{S}$ diminuíram e os de $\mathrm{Fe}$ e $\mathrm{Mg}$ aumentaram linearmente com a adubação nitrogenada. A produtividade de nozes e de amêndoas correlacionou-se positivamente com o teor de $\mathrm{N}$ nas folhas, e a faixa de 14 a $18 \mathrm{~g} \mathrm{~kg}^{-1}$ foi a que possibilitou as maiores produtividades. A dose de $150 \mathrm{~kg} \mathrm{ha}^{-1} \mathrm{de} \mathrm{N}$ por ano proporciona maior produtividade de noz, sem reduzir a taxa de recuperação de amêndoas.

Termos para indexação: Macadamia integrifolia, adubação nitrogenada, amêndoas, nozes, taxa de recuperação, teor foliar.

\section{Nutrition and yield of macadamia nut as affected by nitrogen doses}

\begin{abstract}
The objective of this work was to evaluate the influence of nitrogen doses on mineral nutrition and yield of macadamia nut (Macadamia integrifolia). The experiment was carried out during three agricultural years, in Jaboticabal, SP, Brazil. The experimental design was a complete block with four replicates. Five doses of $\mathrm{N}\left(0,50,100,150\right.$, and $200 \mathrm{~kg} \mathrm{ha}^{-1}$ per year), applied as urea, were evaluated. The increase in $\mathrm{N}$ doses increased the nutrient concentration in the leaves and the yield of nuts and almonds. $\mathrm{Ca}, \mathrm{Mg}$, and $\mathrm{S}$ contents decreased and the ones of $\mathrm{Fe}$ and $\mathrm{Mg}$ increased linearly with $\mathrm{N}$ fertilization. Nut and almond yields were positively correlated with leaf $\mathrm{N}$ concentration, and the range from 14 to $18 \mathrm{~g} \mathrm{~kg}^{-1}$ allowed the highest yields. The dose of $150 \mathrm{~kg} \mathrm{ha}^{-1}$ of $\mathrm{N}$ per year provides higher nut yields, without reducing the recovery rate of almonds.
\end{abstract}

Index terms: Macadamia integrifolia, nitrogen fertilization, almonds, nuts, recovery rate, leaf concentration.

\section{Introdução}

O mercado nacional de noz macadâmia (Macadamia integrifolia Maiden \& Betche) tem crescido rapidamente. As três maiores indústrias estão localizadas nos estados de São Paulo, Espírito Santo e Rio de Janeiro, com capacidade total de beneficiamento de 6 mil toneladas de amêndoas por ano (Sobierajski et al., 2006). O Brasil está entre os países com maior potencial para a produção dessa noz no mundo. Extensas áreas na região Centro-Sul apresentam condições climáticas favoráveis para sua produção (Schneider et al., 2012). Contudo, atualmente, o parque industrial brasileiro opera com apenas metade da capacidade instalada, pela falta da noz como matéria prima.

$\mathrm{O}$ avanço da cultura depende da melhoria na produtividade das lavouras brasileiras que, nos principais estados produtores, é de 10 a $12 \mathrm{~kg}$ por planta por ano, em pomares adultos (Sobierajski et al., 2006; São Paulo, 2008). No entanto, segundo a literatura, uma planta adulta pode produzir $125 \mathrm{~kg}$ de nozes por ano (Pimentel et al., 2007). As baixas produtividades no país podem estar relacionadas à falta de um melhor conhecimento quanto à adubação nitrogenada mais adequada para a cultura (Huett et al., 2001; Olesen, 2005; Fletcher et al., 2010). Para o Estado de São 
Paulo, Quaggio et al. (1997) recomendam $50 \mathrm{~kg} \mathrm{ha}^{-1}$ de $\mathrm{N}$ por ano para lavouras de nogueira-macadâmia com produtividade de até $5 \mathrm{Mg} \mathrm{ha}^{-1}$ por ano, e a faixa considerada ideal para teores foliares de $\mathrm{N}$ é de 15 a $25 \mathrm{~g} \mathrm{~kg}^{-1}$. Porém, não há conhecimento de trabalhos nacionais publicados com recomendações para a espécie. Assim, as tecnologias utilizadas na cultura, no Brasil, são adaptações de pesquisas desenvolvidas na Austrália e nos Estados Unidos (Sacramento \& Pereira, 2003).

Na Austrália, maior produtor mundial, as doses mais utilizadas estão entre 360 e $690 \mathrm{~g}$ de $\mathrm{N}$ por planta por ano, com teores foliares de referência na folha entre 14 e $15 \mathrm{~g} \mathrm{~kg}^{-1}$ de N. No entanto, alguns trabalhos sugerem que há correlação negativa entre produtividade e teor de $\mathrm{N}$ na folha, e que $230 \mathrm{~g}$ de $\mathrm{N}$ por planta por ano, divididos em aplicações mensais, e teores foliares de $12 \mathrm{~g} \mathrm{~kg}^{-1}$ de $\mathrm{N}$ são mais econômicos e eficientes (Stephenson et al., 1997).

Há relatos de que a nogueira-macadâmia necessita de um balanço adequado entre as partes reprodutivas e vegetativas da planta, e de que adubações desequilibradas podem estimular fluxos de vegetação que provocariam o abortamento de frutos e reduziriam a produtividade (Olesen, 2005; McFadyen et al., 2011). Entretanto, Huett et al. (2001) alertam que baixas doses de $\mathrm{N}$ podem gerar, em longo prazo, diminuição da emissão de ramos produtivos e baixa densidade de folhas, o que ocasionaria baixa produtividade nos anos seguintes. Huett \& Vimpany (2007) não encontraram correlação negativa entre doses de $\mathrm{N}$ e produtividade de nozes e relataram teores de até $20 \mathrm{~g} \mathrm{~kg}^{-1}$ de $\mathrm{N}$ na folha, como ideais para a cultura.

O objetivo deste trabalho foi avaliar a influência de doses de $\mathrm{N}$ na nutrição mineral e na produtividade da nogueira-macadâmia.

\section{Material e Métodos}

O experimento foi realizado no Município de Jaboticabal, SP $\left(21^{\circ} 08^{\prime} \mathrm{S}\right.$ e $48^{\circ} 11^{\prime} \mathrm{W}$, a $583 \mathrm{~m}$ de altitude). O clima da região, segundo a classificação de Köppen, é do tipo Aw, tropical com estação seca, com temperatura média anual de $23,2^{\circ} \mathrm{C}$ e precipitação de $1.405 \mathrm{~mm}$ (Tabela 1).

O solo é classificado como Latossolo Vermelho eutrófico, de textura média (Santos et al., 2006). Antes da instalação do experimento, as análises químicas das amostras de solo coletadas na faixa de adubação, na camada de $0-20 \mathrm{~cm}$ de profundidade, indicaram: $18 \mathrm{~g} \mathrm{dm}^{-3}$ de matéria orgânica; $\mathrm{pH}$ em $\mathrm{CaCl}_{2}$ de 5,7; $27 \mathrm{mg} \mathrm{dm}^{-3}$ de P (resina); 1,8, 23, 7 e 49 mmol $_{\mathrm{c}} \mathrm{dm}^{-3}$ de $\mathrm{K}, \mathrm{Ca}, \mathrm{Mg}$ e CTC, respectivamente; saturação por bases de $67 \%$; e $5 \mathrm{mg} \mathrm{dm}{ }^{-3}$ de $\mathrm{S}^{-S}{ }_{4}{ }^{2-}$. Os resultados das análises foliares (Malavolta et al., 1997) por $\mathrm{kg}$ foram: 14,2 $\mathrm{g}$ de $\mathrm{N} ; 0,90 \mathrm{~g}$ de $\mathrm{P} ; 7,5 \mathrm{~g}$ de $\mathrm{K} ; 6,8 \mathrm{~g}$ de $\mathrm{Ca} ; 0,94 \mathrm{~g}$ de $\mathrm{Mg} ; 1,7 \mathrm{~g}$ de $\mathrm{S} ; 4,6 \mathrm{mg}$ de $\mathrm{Cu} ; 98,8 \mathrm{mg}$ de Fe; 316,7 mg de Mn; 21,7 g de B; e 6,7 mg de Zn.

As plantas, da variedade HAES 344 (de origem havaiana), enxertadas sobre porta-enxerto Aloha IAC 10-14 foram plantadas em 1998, no espaçamento $8 \times 6 \mathrm{~m}$. Do plantio até o início do experimento, as adubações foram realizadas conforme Quaggio et al. (1997), tendo-se considerado produtividade esperada de até $5 \mathrm{Mg} \mathrm{ha}^{-1}$, na fase de produção. No período em que se realizou o experimento (de 2008/2009 a $2010 / 2011$ ), as plantas estavam com bom vigor, com produção média de $11,6 \mathrm{~kg}$ por planta $\left(2.417 \mathrm{~kg} \mathrm{ha}^{-1}\right)$, no ano anterior à instalação dos experimentos (2007/2008). Essa produção é considerada adequada pelos produtores do estado, para a idade do pomar.

O experimento foi iniciado em outubro de 2008. Utilizou-se o delineamento de blocos ao acaso com quatro repetições. Os tratamentos consistiram de cinco doses de $\mathrm{N}\left(0,50,100,150\right.$ e $\left.200 \mathrm{~kg} \mathrm{ha}^{-1}\right)$, múltiplas da dose de $50 \mathrm{~kg} \mathrm{ha}^{-1}$ por ano recomendada por Quaggio et al. (1997). A fonte de $\mathrm{N}$ utilizada foi a ureia. Cada unidade experimental foi composta por sete plantas, e a área útil foi composta pelas cinco plantas centrais, num total de $240 \mathrm{~m}^{2}$. Em todos os tratamentos, a aplicação de $\mathrm{N}$ foi fracionada em três parcelas iguais, em outubro, dezembro e fevereiro de cada ano, e distribuída uniformemente sob a copa das plantas. Para garantir o suprimento de potássio e fósforo, foram aplicadas doses acima das recomendadas por Quaggio et al. (1997), em todas as unidades experimentais, ou seja, $100 \mathrm{~kg} \mathrm{ha}^{-1}$ de $\mathrm{K}_{2} \mathrm{O}$ (cloreto de potássio), parcelados nas mesmas épocas da adubação nitrogenada, e $50 \mathrm{~kg} \mathrm{ha}^{-1}$ de $\mathrm{P}_{2} \mathrm{O}_{5}$ (superfosfato simples), aplicados em uma única dose, em outubro de cada ano.

Foram realizadas três adubações foliares (outubro, dezembro e fevereiro), com pulverizações de 1,8 $\mathrm{kg} \mathrm{ha}^{-1}$ de $\mathrm{N}$ (ureia), 1,2 $\mathrm{kg} \mathrm{ha}^{-1}$ de $\mathrm{Cu}$ (oxicloreto de cobre), $0,48 \mathrm{~kg} \mathrm{ha}^{-1}$ de $\mathrm{Zn}$ (sulfato de zinco) e $0,2 \mathrm{~kg} \mathrm{ha}^{-1}$ de B (ácido bórico). As plantas daninhas foram controladas com três roçadas mecanizadas nas entrelinhas e uma 
aplicação do herbicida glifosato ( $720 \mathrm{~g} \mathrm{ha}^{-1}$ do i.a.), em área total para o preparo da colheita.

Em setembro de cada ano, foram coletadas folhas (terceira folha madura) de ramos posicionados no terço médio das plantas, nas quatro posições cardeais, num total de 100 folhas por parcela (Stephenson et al., 1997). As folhas foram submetidas à lavagem com água destilada, acondicionadas em sacos de papel, levadas à estufa com circulação forçada de ar a 60-70 ${ }^{\circ} \mathrm{C}$ para secagem e, em seguida, moídas para análise do teor de nutrientes $(\mathrm{N}, \mathrm{P}, \mathrm{K}, \mathrm{Ca}, \mathrm{Mg}, \mathrm{S}, \mathrm{B}$, $\mathrm{Cu}, \mathrm{Fe}, \mathrm{Mn}$ e $\mathrm{Zn}$ ), segundo metodologia descrita por Malavolta et al. (1997).

Os frutos maduros foram coletados no chão, entre fevereiro e maio de cada ano. Após a remoção dos carpelos, os frutos foram secos à sombra, até atingirem teor de água entre 10 e $11 \%$, e pesados para determinação da produtividade de nozes (casca + amêndoa) por planta. Também foram determinados: número de nozes por planta; peso médio de uma noz (a partir do peso de 100 nozes); peso médio de uma amêndoa (mediante a quebra, limpeza e pesagem das amêndoas, de uma amostra de 100 nozes); taxa de recuperação de amêndoas (percentagem de amêndoa em relação ao peso total da noz); e produtividade de amêndoas por planta (mediante a multiplicação da produção de nozes pela taxa de recuperação de amêndoas).

Os dados foram submetidos à análise de variância, com análise conjunta dos dados dos três anos. Para as variáveis que não apresentaram efeito significativo da interação entre doses de $\mathrm{N}$ e anos, foram apresentados

Tabela 1. Precipitação e temperatura média, mensais, no período de julho de 2008 a junho de 2011.

\begin{tabular}{|c|c|c|c|c|c|c|}
\hline \multirow[t]{2}{*}{ Mês } & \multicolumn{3}{|c|}{ Precipitação (mm) } & \multicolumn{3}{|c|}{ Temperatura média $\left({ }^{\circ} \mathrm{C}\right)$} \\
\hline & $\begin{array}{l}2008 / \\
2009\end{array}$ & $\begin{array}{l}2009 / \\
2010\end{array}$ & $\begin{array}{c}2010 / \\
2011\end{array}$ & $\begin{array}{c}2008 / \\
2009\end{array}$ & $\begin{array}{c}2009 / \\
2010\end{array}$ & $\begin{array}{c}2010 / \\
2011\end{array}$ \\
\hline Julho & 0,0 & 25,5 & 0,0 & 20,3 & 21,0 & 21,5 \\
\hline Agosto & 24,2 & 133,1 & 0,0 & 22,7 & 21,3 & 21,8 \\
\hline Setembro & 15,1 & 131,5 & 141,9 & 22,5 & 23,6 & 24,2 \\
\hline Outubro & 60,5 & 101,9 & 69,4 & 25,4 & 24,5 & 23,8 \\
\hline Novembro & 81,8 & 163,3 & 100,1 & 25,5 & 26,6 & 24,9 \\
\hline Dezembro & 278,9 & 383,7 & 225,3 & 25,1 & 25,2 & 26,0 \\
\hline Janeiro & 238,0 & 240,7 & 260,1 & 24,8 & 25,6 & 26,0 \\
\hline Fevereiro & 190,6 & 150,7 & 208,2 & 25,9 & 26,3 & 26,1 \\
\hline Março & 217,9 & 183,0 & 496,0 & 25,6 & 25,7 & 24,1 \\
\hline Abril & 70,8 & 95,5 & 92,3 & 23,3 & 23,2 & 23,8 \\
\hline Maio & 26,6 & 10,6 & 7,0 & 21,9 & 20,6 & 20,8 \\
\hline Junho & 51,9 & 7,8 & 29,7 & 18,6 & 19,7 & 19,0 \\
\hline
\end{tabular}

apenas os resultados médios dos três anos. Os efeitos das doses de $\mathrm{N}$ foram avaliados por meio de análise de regressão, tendo-se utilizado, como critério para escolha do modelo, a magnitude do $\mathrm{R}^{2}$ das equações com coeficientes de regressão significativos pelo teste $\mathrm{t}$, a $5 \%$ de probabilidade.

\section{Resultados e Discussão}

$\mathrm{O}$ teor de $\mathrm{N}$ na folha aumentou em função da adubação nitrogenada, nos três anos de experimentação (Tabela 2). Nos dois primeiros anos, o aumento das doses de $\mathrm{N}$ aplicadas proporcionou incrementos lineares no teor do nutriente na folha. No terceiro ano, a resposta foi quadrática, com o máximo teor obtido com a dose estimada de $186 \mathrm{~kg} \mathrm{ha}^{-1}$ de N. As doses de $50 \mathrm{~kg} \mathrm{ha}^{-1}$ de $\mathrm{N}$, recomendadas por Quaggio et al. (1997), para produtividade esperada de até $5 \mathrm{Mg}$, não foram suficientes, nas duas primeiras safras e na média das safras, para elevar os teores de $\mathrm{N}$ na folha a valores considerados adequados pelos autores, entre 15 e $25 \mathrm{~g} \mathrm{~kg}^{-1}$.

No ano agrícola 2008/2009, esses teores não foram alcançados com nenhuma das doses de $\mathrm{N}$ avaliadas. Em 2009/2010, eles foram alcançados somente com doses acima de $85 \mathrm{~kg} \mathrm{ha}^{-1}$ e, em 2010/2011, com a dose de $60 \mathrm{~kg} \mathrm{ha}^{-1}$ (Tabela 2). As menores precipitações pluviais nos meses que precederam a coleta de folhas, nos anos agrícolas 2008/2009 e 2010/2011, podem explicar os menores teores de $\mathrm{N}$ nas folhas em relação ao ano agrícola 2009/2010, especialmente nas maiores doses de N (Tabelas 1 e 2). Ao se considerar a média dos anos, doses acima de $90 \mathrm{~kg} \mathrm{ha}^{-1}$ foram suficientes para proporcionar valores dentro da faixa considerada adequada por Quaggio et al. (1997). Adubações inferiores a $50 \mathrm{~kg} \mathrm{ha}^{-1}$ de $\mathrm{N}$ foram suficientes para manter os valores de $\mathrm{N}$ na folha dentro da faixa ideal de 13 a $14 \mathrm{~g} \mathrm{~kg}^{-1}$, adotada por Reuter \& Robinson (1997), e adubações entre 50 e $100 \mathrm{~kg} \mathrm{ha}^{-1}$ foram eficientes para elevar os teores para valores entre 14 e $15 \mathrm{~g} \mathrm{~kg}^{-1}$, propostos por Stephenson \& Cull (1986). Somente doses acima de $100 \mathrm{~kg} \mathrm{ha}^{-1}$ adequaram os teores foliares de $\mathrm{N}$ aos valores entre 16 e $20 \mathrm{~g} \mathrm{~kg}^{-1}$, sugeridos por Huett \& Vimpany (2007) para a cultivar HAES 344. Dessa forma, em razão das divergências entre os autores, as doses necessárias de $\mathrm{N}$ para atingir teores foliares adequados estariam entre 45 e $200 \mathrm{~kg} \mathrm{ha}^{-1}$, o que representa uma variação muito elevada e reforça a 
importância da realização de estudos específicos para as diversas regiões de cultivo e sistemas de produção utilizados.

$\mathrm{Na}$ média dos três anos, os teores de $\mathrm{P}, \mathrm{K}, \mathrm{B}, \mathrm{Cu}$ e $\mathrm{Zn}$ (elementos que foram fornecidos via solo ou folha) não foram afetados pelas doses de $\mathrm{N}$ (Tabela 3 ). Os teores de $\mathrm{Ca}, \mathrm{Mg}$ e $\mathrm{S}$ diminuíram linearmente com a aplicação de $\mathrm{N}$; já os teores de $\mathrm{Fe}$ e $\mathrm{Mg}$ aumentaram. Esses resultados estão provavelmente relacionados à acidificação que a aplicação de $\mathrm{N}$ provoca no solo (Stephenson et al., 1997). Os teores de P na folha variaram entre 0,91 e $0,95 \mathrm{~g} \mathrm{~kg}^{-1}$, valores dentro das faixas adequadas sugeridas por Stephenson \& Cull (1986), Reuter \& Robinson (1997) e Huett \& Vimpany (2007), mas abaixo do proposto por Quaggio et al. (1997). Os teores de K $\left(7,9-8,1 \mathrm{~kg}^{-1}\right)$ estavam dentro da faixa recomendada por Quaggio et al. (1997), no limite superior das de Reuter \& Robinson (1997) e Huett \& Vimpany (2007), porém acima da faixa sugerida por Stephenson \& Cull (1986). Os teores foliares de Ca, Fe e Mn estavam dentro das faixas propostas por todos esses autores, enquanto os teores de $\mathrm{Mg}$ ficaram abaixo das faixas consideradas adequadas. Os teores de $\mathrm{S}$ estavam dentro das faixas consideradas adequadas por Quaggio et al. (1997) e Huett \& Vimpany (2007). Os teores de B estavam dentro do adequado, de acordo com Quaggio et al. (1997), mas abaixo das demais faixas, enquanto os teores de $\mathrm{Cu}$ estavam acima das faixas adequadas.

$\mathrm{O}$ número de nozes por planta aumentou de forma linear com o aumento nas doses de $\mathrm{N}$, em todos os anos (Tabela 4). Stephenson \& Cull (1986) observaram que, sem suplementação de N, plantas de nogueira-macadâmia têm baixo desenvolvimento do caule e de ramos, além de baixa densidade de folhas, o que tem efeito negativo na produtividade da cultura. Stephenson \& Gallagher (1989) constataram menor floração em plantas que receberam $230 \mathrm{~g}$ de $\mathrm{N}$ do que nas que receberam $690 \mathrm{~g}$ por ano. No entanto, Fletcher et al. (2010), em estudo com ${ }^{15} \mathrm{~N}$, concluíram que o $\mathrm{N}$ proveniente do solo é inicialmente distribuído para folhas jovens e maduras - que se tornam fontes de $\mathrm{N}$ para flores e frutos na primavera seguinte - e que não há competição por fotoassimilados entre brotos novos e frutos. McFadyen et al. (2011) consideram que, nos estádios iniciais, os frutos da nogueira-macadâmia não representam um forte dreno de fotoassimilados e são vulneráveis à competição por carboidratos com as

Tabela 2. Teores foliares de $\mathrm{N}\left(\mathrm{g} \mathrm{kg}^{-1}\right)$ em nogueira-macadâmia (Macadamia integrifolia) em função das doses de nitrogênio.

\begin{tabular}{|c|c|c|c|c|c|c|c|c|}
\hline \multirow[t]{2}{*}{ Ano agrícola } & \multicolumn{5}{|c|}{ Dose de $\mathrm{N}\left(\mathrm{kg} \mathrm{ha}^{-1}\right)$} & \multirow{2}{*}{$\begin{array}{l}\text { CV } \\
(\%)\end{array}$} & \multirow[t]{2}{*}{ Equação de regressão } & \multirow[t]{2}{*}{$\mathrm{R}^{2}$} \\
\hline & 0 & 50 & 100 & 150 & 200 & & & \\
\hline $2008 / 2009$ & 12,6 & 13,8 & 13,7 & 14,2 & 14,7 & 4,8 & $\mathrm{y}=12,82+0,00954 * *^{\mathrm{x}}$ & 0,89 \\
\hline $2009 / 2010$ & 12,4 & 13,5 & 16,3 & 16,8 & 18,2 & 5,8 & $\mathrm{y}=12,48+0,029680 * *_{\mathrm{x}}$ & 0,95 \\
\hline $2010 / 2011$ & 12,6 & 15,3 & 15,9 & 16,2 & 17,1 & 3,7 & $\mathrm{y}=12,91+0,0409 * * \mathrm{x}-0,00011 * * \mathrm{x}^{2}$ & 0,94 \\
\hline Média & 12,5 & 14,2 & 15,3 & 15,7 & 16,7 & 2,2 & $\mathrm{y}=12,61+0,0315 * * x-0,000059 * * x^{2}$ & 0,99 \\
\hline
\end{tabular}

**Significativo pelo teste $\mathrm{t}$, a $1 \%$ de probabilidade.

Tabela 3. Teores foliares de nutrientes (P, K, Ca, Mg, S, B, Cu, Fe, Mn e Zn) em nogueira-macadâmia (Macadamia integrifolia), em função das doses de nitrogênio ${ }^{(1)}$.

\begin{tabular}{|c|c|c|c|c|c|c|c|c|}
\hline \multirow[t]{2}{*}{ Nutriente } & \multicolumn{5}{|c|}{ Dose de $\mathrm{N}\left(\mathrm{kg} \mathrm{ha}^{-1}\right)$} & \multirow{2}{*}{$\begin{array}{l}\mathrm{CV} \\
(\%)\end{array}$} & \multirow[t]{2}{*}{ Equação de regressão } & \multirow[t]{2}{*}{$\mathrm{R}^{2}$} \\
\hline & 0 & 50 & 100 & 150 & 200 & & & \\
\hline $\mathrm{P}\left(\mathrm{g} \mathrm{kg}^{-1}\right)$ & 0,91 & 0,91 & 0,94 & 0,93 & 0,95 & 5,4 & ns & - \\
\hline $\mathrm{K}\left(\mathrm{g} \mathrm{kg}^{-1}\right)$ & 8,1 & 7,9 & 7,9 & 8,0 & 7,8 & 5,6 & ns & - \\
\hline $\mathrm{Ca}\left(\mathrm{g} \mathrm{kg}^{-1}\right)$ & 6,6 & 5,9 & 6,0 & 6,1 & 5,7 & 5,0 & $\mathrm{y}=6,40-0,003287 * * x$ & 0,63 \\
\hline $\operatorname{Mg}\left(\mathrm{g} \mathrm{kg}^{-1}\right)$ & 0,75 & 0,72 & 0,71 & 0,67 & 0,68 & 6,9 & $y=0,7436-0,000379 * x$ & 0,91 \\
\hline $\mathrm{S}\left(\mathrm{g} \mathrm{kg}^{-1}\right)$ & 1,2 & 1,2 & 1,2 & 1,1 & 1,1 & 6,1 & $\mathrm{y}=1,2223-0,000497 *^{*} \mathrm{x}$ & 0,74 \\
\hline $\mathrm{B}\left(\mathrm{mg} \mathrm{kg}^{-1}\right)$ & 31,3 & 42,3 & 36,8 & 36,8 & 32,1 & 27,0 & ns & - \\
\hline $\mathrm{Cu}\left(\mathrm{mg} \mathrm{kg}^{-1}\right)$ & 12,8 & 24,4 & 16,1 & 12,1 & 11,1 & 39,4 & ns & - \\
\hline $\mathrm{Fe}\left(\mathrm{mg} \mathrm{kg}^{-1}\right)$ & 82,4 & 84,4 & 92,0 & 94,9 & 100,9 & 11,7 & $\mathrm{y}=81,45+0,094648 * x$ & 0,97 \\
\hline $\operatorname{Mn}\left(\mathrm{mg} \mathrm{kg}^{-1}\right)$ & 329,2 & 404,4 & 451,3 & 470,2 & 521,4 & 20,2 & $\mathrm{y}=345,25+0,9005 * * \mathrm{x}$ & 0,96 \\
\hline $\mathrm{Zn}\left(\mathrm{mg} \mathrm{kg}^{-1}\right)$ & 10,7 & 9,5 & 8,9 & 9,3 & 8,4 & 13,7 & ns & - \\
\hline
\end{tabular}

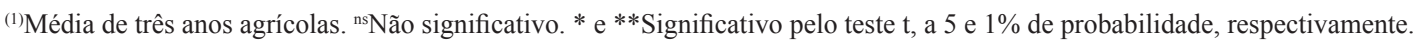


zonas de crescimento vegetativo, enquanto Stephenson et al. (1997) obtiveram maiores produtividades em plantas com menores teores de $\mathrm{N}$ nas folhas $\left(12 \mathrm{~g} \mathrm{~kg}^{-1}\right)$. No presente trabalho, houve correlação positiva entre o teor foliar de $\mathrm{N}$ e o número de nozes por planta, em todos os anos (Tabela 5).

$\mathrm{O} \mathrm{N}$ é necessário para atender à demanda de fotoassimilados dos frutos (Stephenson \& Cull, 1986); porém, praticamente não houve interferência das doses de N no peso médio da noz, com exceção do ano agrícola 2010/2011, no qual as doses de $\mathrm{N}$ proporcionaram leve aumento nessa variável, com valores máximos obtidos com a dose estimada de $102 \mathrm{~kg} \mathrm{ha}^{-2}$ de $\mathrm{N}$ (Tabela 4). Entretanto, constatou-se ausência de correlação entre essa variável e o teor de $\mathrm{N}$ na folha, em todos os anos agrícolas (Tabela 5). Stephenson et al. (2002) observaram maior percentagem de nozes não comercializáveis (menores que $19 \mathrm{~mm}$ ) com o uso de maiores doses de $\mathrm{N}$.

A produtividade de nozes aumentou linearmente com as doses de $\mathrm{N}$ aplicadas (Tabela 4) e correlacionouse positivamente com o teor de $\mathrm{N}$ na folha (Tabela 5), em todos os anos de estudo. Esses resultados diferem dos obtidos por Stephenson et al. (1997) que, ao testar doses de 230, 690 e 1.150 g de N por planta, encontraram correlação negativa entre o teor de $\mathrm{N}$ nas folhas e a produtividade de nozes. Os autores atribuíram essa relação negativa à competição entre o desenvolvimento vegetativo e a produção. Huett \& Vimpany (2007), no entanto, não constataram

Tabela 4. Número médio de nozes por planta, peso médio da noz, produtividade de nozes, peso médio da amêndoa, taxa de recuperação e produtividade de amêndoas de nogueira-macadâmia (Macadamia integrifolia) em função de doses de nitrogênio.

\begin{tabular}{|c|c|c|c|c|c|c|c|c|}
\hline \multirow[t]{2}{*}{ Ano agrícola } & \multicolumn{5}{|c|}{ Dose de $\mathrm{N}\left(\mathrm{kg} \mathrm{ha}^{-1}\right)$} & \multirow{2}{*}{$\begin{array}{l}\mathrm{CV} \\
(\%)\end{array}$} & \multirow{2}{*}{ Equação de regressão } & \multirow{2}{*}{$\mathrm{R}^{2}$} \\
\hline & 0 & 50 & 100 & 150 & 200 & & & \\
\hline \multicolumn{9}{|c|}{ Número de nozes por planta } \\
\hline $2008 / 2009$ & 2.085 & 2.477 & 2.706 & 2.658 & 2.887 & 12,1 & $\mathrm{y}=2.205,6+3,57 * * x$ & 0,86 \\
\hline $2009 / 2010$ & 1.994 & 2.214 & 2.451 & 2.411 & 2.677 & 12,4 & $\mathrm{y}=2.036,7+3,128 * * x$ & 0,92 \\
\hline $2010 / 2011$ & 785 & 971 & 1.096 & 1.228 & 1.140 & 22,1 & $\mathrm{y}=850,4+1,932 * \mathrm{x}$ & 0,79 \\
\hline Média & 1.621 & 1.887 & 2.084 & 2.099 & 2.235 & 6,2 & $\mathrm{y}=1.697,6+2,877 * * \mathrm{x}$ & 0,91 \\
\hline \multicolumn{9}{|c|}{ Peso médio da noz $(\mathrm{g})$} \\
\hline $2008 / 2009$ & 7,5 & 7,5 & 7,8 & 7,9 & 7,6 & 4,0 & ns & - \\
\hline $2009 / 2010$ & 7,4 & 7,9 & 7,5 & 7,6 & 8,0 & 5,5 & ns & - \\
\hline $2010 / 2011$ & 7,5 & 7,8 & 8,0 & 7,6 & 7,6 & 3,3 & $y=7,51+0,0067 * x-0,000033 * x^{2}$ & 0,56 \\
\hline Média & 7,5 & 7,8 & 7,8 & 7,7 & 7,7 & 2,7 & ns & - \\
\hline \multicolumn{9}{|c|}{ Produtividade de nozes (kg por planta) } \\
\hline $2008 / 2009$ & 15,7 & 18,6 & 21,2 & 21,1 & 22,0 & 10,4 & $\mathrm{y}=16,743+0,02972 * * \mathrm{x}$ & 0,85 \\
\hline $2009 / 2010$ & 14,8 & 17,3 & 18,5 & 18,4 & 21,3 & 11,5 & $\mathrm{y}=15,237+0,02814 * * x$ & 0,91 \\
\hline $2010 / 2011$ & 5,9 & 7,6 & 8,7 & 9,3 & 8,7 & 20,4 & $\mathrm{y}=6,550+0,01450 * \mathrm{x}$ & 0,73 \\
\hline Média & 12,1 & 14,5 & 16,1 & 16,2 & 17,3 & 6,2 & $\mathrm{y}=12,25+0,0476 * * x-0,00012 * x^{2}$ & 0,97 \\
\hline \multicolumn{9}{|c|}{ Peso médio da amêndoa $(\mathrm{g})$} \\
\hline $2008 / 2009$ & 2,0 & 2,0 & 2,1 & 2,2 & 2,0 & 7,2 & ns & - \\
\hline $2009 / 2010$ & 1,9 & 2,2 & 2,1 & 2,0 & 2,0 & 6,2 & $\mathrm{y}=1,95+0,0035^{*} \mathrm{x}-0,000017 * \mathrm{x}^{2}$ & 0,36 \\
\hline $2010 / 2011$ & 1,9 & 2,2 & 2,3 & 2,0 & 2,0 & 10,0 & $\mathrm{y}=1,91+0,00650 * \mathrm{x}-0,000032 * \mathrm{x}^{2}$ & 0,68 \\
\hline Média & 1,9 & 2,1 & 2,2 & 2,1 & 2,0 & 2,8 & $\mathrm{y}=1,94+0,00420 * \mathrm{x}-0,000020 * \mathrm{x}^{2}$ & 0,82 \\
\hline \multicolumn{9}{|c|}{ Taxa de recuperação (\%) } \\
\hline $2008 / 2009$ & 26,6 & 26,6 & 27,1 & 27,5 & 26,4 & 6,3 & ns & - \\
\hline $2009 / 2010$ & 25,3 & 28,4 & 27,9 & 26,3 & 25,7 & 6,7 & $\mathrm{y}=25,76+0,0461 * x-0,000244 * x^{2}$ & 0,72 \\
\hline $2010 / 2011$ & 25,2 & 27,6 & 29,5 & 26,5 & 26,2 & 8,4 & $\mathrm{y}=25,35+0,0605 * \mathrm{x}-0,000294 * \mathrm{x}^{2}$ & 0,72 \\
\hline Média & 25,7 & 27,5 & 28,5 & 28,2 & 26,8 & 26,1 & $\mathrm{y}=25,83+0,0405 * * x-0,000203 * * x^{2}$ & 0,87 \\
\hline \multicolumn{9}{|c|}{ Produtividade de amêndoas (kg por planta) } \\
\hline $2008 / 2009$ & 4,2 & 5,0 & 5,8 & 5,8 & 5,8 & 12,6 & $\mathrm{y}=4,49+0,008175^{* *} \mathrm{x}$ & 0,80 \\
\hline $2009 / 2010$ & 3,7 & 4,9 & 5,1 & 4,8 & 5,5 & 12,8 & $\mathrm{y}=4,15+0,00668^{* *} \mathrm{x}$ & 0,65 \\
\hline $2010 / 2011$ & 1,5 & 2,1 & 2,5 & 2,5 & 2,5 & 2,3 & $\mathrm{y}=1,48+0,015652 * * x-0,000059 * x^{2}$ & 0,99 \\
\hline Média & 3,1 & 4,0 & 4,5 & 4,4 & 4,5 & 7,1 & $\mathrm{y}=3,16+0,01865 * * \mathrm{x}-0,000062 * * \mathrm{x}^{2}$ & 0,94 \\
\hline
\end{tabular}

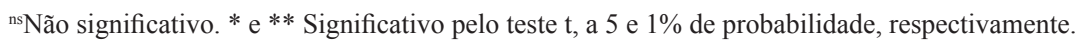


correlação negativa entre doses de $\mathrm{N}$ e produtividade de nozes, e Fletcher et al. (2010) destacaram que o N absorvido pela nogueira-macadâmia é inicialmente distribuído para folhas jovens que, ao amadurecerem, tornam-se fontes de $\mathrm{N}$ para flores e frutos, o que poderia explicar os resultados obtidos no presente trabalho.

A baixa precipitação pluvial ocorrida nos meses anteriores ao mês do florescimento (agosto), no ano agrícola 2010/2011 (Tabela 1), causou a seca de muitas flores e o abortamento de frutos jovens, o que diminuiu sensivelmente o número de nozes por planta e a produtividade e pode ter limitado a resposta ao $\mathrm{N}$ aplicado (Tabela 4). Nesse mesmo ano agrícola, o aumento do peso médio da noz com a aplicação de $\mathrm{N}$ pode estar relacionado ao menor número de frutos retidos nas plantas. Segundo Stephenson et al. (2002), plantas de nogueira-macadâmia com grande número de frutos podem produzir frutos de menor tamanho e peso. Trochoulias \& Johns (1992) relataram que a irrigação da nogueira-macadâmia reduziu o tamanho da noz, em decorrência da retenção de mais frutos por planta.

O peso médio da amêndoa foi afetado pelas doses de N apenas nos anos agrícolas 2009/2010 e 2010/2011, com os dados ajustados a equações quadráticas. Os valores máximos foram obtidos com doses estimadas de aproximadamente $105 \mathrm{~kg} \mathrm{ha}^{-1} \mathrm{de}$ $\mathrm{N}$ (Tabela 4). As variações do peso das amêndoas refletiram-se na taxa de recuperação, que apresentou resposta semelhante a essa variável. Contudo, as variações nos valores de ambas as variáveis foram pequenas e não houve correlação destas com o teor de $\mathrm{N}$ na folha (Tabela 5). Penoni et al. (2011) obtiveram taxa de recuperação um pouco superior com a cultivar HAES 344 (31\%), provavelmente por terem efetuado as medições logo após a colheita, sem terem secado as nozes. Sabe-se que as indústrias pagam ágios por lotes com maiores taxas de recuperação, em razão do maior rendimento no processamento. Portanto, a quantidade de nozes produzidas é importante, mas a qualidade do produto, representada pela taxa de recuperação, é fator decisivo na formação do preço e na lucratividade do investimento (Pimentel et al., 2007). A produtividade de amêndoas foi linearmente influenciada pelas doses de $\mathrm{N}$ nos anos agrícolas 2008/2009 e 2009/2010, e de forma quadrática em 2010/2011, com ponto de máxima estimado em $155 \mathrm{~kg} \mathrm{ha}^{-1}$ de N (Tabela 4).

$\mathrm{Na}$ África do Sul, terceiro maior produtor mundial da noz, recomenda-se a aplicação ao solo de $360 \mathrm{~g}$ de $\mathrm{N}$ por planta por ano (72 a $\left.90 \mathrm{~kg} \mathrm{ha}^{-1}\right)$, em pomares adultos, e os níveis ideais de $\mathrm{N}$ na folha ficam entre 13 e $15 \mathrm{~g} \mathrm{~kg}^{-1}$ (Manson \& Sheard, 2007). Na Austrália, produtores utilizam rotineiramente $150 \mathrm{~kg} \mathrm{ha}^{-1}$ de N. De acordo com Reuter \& Robinson (1997), os teores foliares ideais são de 13 a $14 \mathrm{~g} \mathrm{~kg}^{-1}$, mas os autores recomendam doses de $\mathrm{N}$ menores. Huett \& Vimpany (2007) concordam com essa adubação e admitem teores foliares entre 14 e $20 \mathrm{~g} \mathrm{~kg}^{-1}$. No Brasil, a recomendação é de $50 \mathrm{~kg} \mathrm{ha}^{-1}$ de $\mathrm{N}$, para produção de até $5 \mathrm{Mg} \mathrm{ha}^{-1}$ (Quaggio et al., 1997), e a maioria dos produtores, no intuito de atingir valores de $\mathrm{N}$ na folha entre 13 e $14 \mathrm{~g} \mathrm{~kg}^{-1}$, recomendadas por Reuter \& Robinson (1997), utilizam doses inferiores a $50 \mathrm{~kg} \mathrm{ha}^{-1}$ de $\mathrm{N}$ ou até mesmo deixam de fazer adubações nitrogenadas em suas lavouras. Como resultado, observa-se produtividade média baixa e muito aquém da capacidade produtiva da espécie (Pimentel et al., 2007). No presente trabalho, a produtividade de nozes foi acrescida em 5,2 kg por planta ou $1.083 \mathrm{~kg} \mathrm{ha}^{-1}$, na média dos anos agrícolas, com uso das maiores doses de $\mathrm{N}$, em comparação ao não uso do $\mathrm{N}$, e em $2,8 \mathrm{~kg}$ por planta ou $583 \mathrm{~kg} \mathrm{ha}^{-1} \mathrm{em}$ comparação à atual dose recomendada.

A dose de $200 \mathrm{~kg} \mathrm{ha}^{-1}$ de $\mathrm{N}$ proporcionou ligeiro acréscimo na produtividade de nozes em comparação à dose de $150 \mathrm{~kg} \mathrm{ha}^{-1}$. E mesmo essas doses elevadas

Tabela 5. Coeficiente de correlação simples entre teor foliar de $\mathrm{N}$ e número médio de nozes por planta, peso médio da noz, produtividade de nozes, peso médio da amêndoa, taxa de recuperação e produtividade de amêndoas de nogueira-macadâmia (Macadamia integrifolia).

\begin{tabular}{|c|c|c|c|c|c|c|}
\hline Variável & $\begin{array}{c}\text { Nozes por } \\
\text { planta }\end{array}$ & $\begin{array}{c}\text { Peso médio } \\
\text { da noz }\end{array}$ & $\begin{array}{c}\text { Produtividade } \\
\text { de nozes }\end{array}$ & $\begin{array}{l}\text { Peso médio } \\
\text { da amêndoa }\end{array}$ & $\begin{array}{c}\text { Taxa de } \\
\text { recuperação }\end{array}$ & $\begin{array}{l}\text { Produtividade } \\
\text { de amêndoas }\end{array}$ \\
\hline Teor de N (2008/2009) & $0,67 * *$ & $-0,02^{\mathrm{ns}}$ & $0,65^{* *}$ & $0,14^{\mathrm{ns}}$ & $0,18^{\mathrm{ns}}$ & $0,63 * *$ \\
\hline Teor de N (2009/2010) & $0,64 * *$ & $0,10^{\mathrm{ns}}$ & $0,67 * *$ & $0,03^{\text {ns }}$ & $-0,03^{\mathrm{ns}}$ & $0,58 * *$ \\
\hline Teor de N (2010/2011) & $0,58 * *$ & $0,25^{\mathrm{ns}}$ & $0,64 * *$ & $0,21^{\mathrm{ns}}$ & $0,17^{\mathrm{ns}}$ & $0,64 * *$ \\
\hline
\end{tabular}

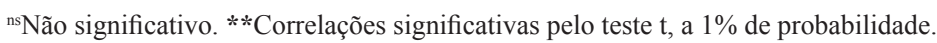


não afetaram a qualidade das amêndoas (Tabela 4). $\mathrm{O}$ teor de $\mathrm{N}$ na folha associado a elas ficou entre $14 \mathrm{e}$ $18 \mathrm{~g} \mathrm{~kg}^{-1}$ (Tabela 2). Esses resultados evidenciam que há a necessidade de revisar as atuais recomendações de adubação e o teor adequado de $\mathrm{N}$ nas folhas para $\mathrm{o}$ cultivo da nogueira-macadâmia no Brasil.

\section{Conclusões}

1. O teor foliar de $\mathrm{N}$ e a produtividade de nozes e amêndoas da nogueira-macadâmia aumentam linearmente com a adubação nitrogenada.

2. Os teores de $\mathrm{Ca}, \mathrm{Mg}$ e $\mathrm{S}$ diminuem e os de $\mathrm{Fe}$ e $\mathrm{Mg}$ aumentam linearmente com a adubação nitrogenada.

3. A produtividade de nozes e de amêndoas correlaciona-se positivamente com o teor de $\mathrm{N}$ nas folhas, e o teor foliar de $\mathrm{N}$ relacionado às maiores produtividades está entre 14 e $18 \mathrm{~g} \mathrm{~kg}^{-1}$.

4. A dose de $150 \mathrm{~kg} \mathrm{ha}^{-1}$ de $\mathrm{N}$ por ano é a mais adequada para a produtividade de noz, e não reduz a taxa de recuperação de amêndoas.

\section{Referências}

FLETCHER, A.; RENNENBERG, H.; SCHMIDT, S. Nitrogen partitioning in orchard-grown Macadamia integrifolia. Tree Physiology, v.30, p.244-256, 2010. DOI: 10.1093/treephys/tpp107.

HUETT, D.O.; GOGEL, B.J.; MEYERS, N.M.; MCCONCHIE, C.A.; MCFADYEN, L.M.; MORRIS, S.C. Leaf nitrogen and phosphorus levels in macadamias in response to canopy position and light exposure, their potential as leaf-based shading indicators, and implications for diagnostic leaf sampling protocols. Australian Journal of Agricultural Research, v.52, p.513-522, 2001. DOI: 10.1071/AR00066.

HUETT, D.O.; VIMPANY, I. Revised diagnostic leaf nutrient standards for macadamia growing in Australia. Australian Journal of Experimental Agriculture, v.47, p.869-876, 2007. DOI: 10.1071/EA06133.

MALAVOLTA, E.; VITTI, G.C.; OLIVEIRA, S.A. de (Ed.). Avaliação do estado nutricional de plantas: princípios e aplicações. 2.ed. Piracicaba: Potafos, 1997. 319p.

MANSON, A.D.; SHEARD, A. Macadamia fertilization in Kwazulu-Natal. Kwazulu-Natal: Department of Agriculture and Environmental Affairs, 2007. 11p. (KZN Agri-report N/A/2007/10.).

MCFADYEN, L.M.; ROBERTSON, D.; SEDGLEY, M.; KRISTIANSEN, P.; OLESEN, T. Post-pruning shoot growth increases fruit abscission and reduces stem carbohydrates and yield in macadamia. Annals of Botany, v.107, p.993-1001, 2011. DOI: 10.1093/aob/mcr026.

OLESEN, T. The timing of flush development affects the flowering of avocado (Persea americana) and macadamia (Macadamia integrifolia $x$ tetraphylla). Australian Journal of Agricultural Research, v.56, p.723-729, 2005. DOI: 10.1071/AR04287.

PENONI, E. dos S.; PIO, R.; RODRIGUES, F.A.; MARO, L.A.C.; COSTA, F.C. Análise de frutos e nozes de cultivares de nogueira-macadâmia. Ciência Rural, v.41, p.2080-2083, 2011. DOI: $10.1590 / \mathrm{S} 0103-84782011001200007$.

PIMENTEL, L.D.; SANTOS, C.E.M. dos; WAGNER JÚNIOR, A.; SILVA, V.A.; BRUCKNER, C.H. Estudo de viabilidade econômica na cultura da noz-macadâmia no Brasil. Revista Brasileira de Fruticultura, v.29, p.500-507, 2007. DOI: 10.1590/ S0100-29452007000300018.

QUAGGIO, J.A.; RAIJ, B. van; PIZA JUNIOR, C.T. Frutíferas. In: RAIJ, B. van; CANTARELLA, H.; QUAGGIO, A.J.; FURLANI, A.M.C. (Ed.). Recomendações de adubação e calagem para o Estado de São Paulo. 2.ed. Campinas: IAC, 1997. p.121-153. (IAC. Boletim técnico, 100).

REUTER, D.J.; ROBINSON, J.B. Plant analysis: an interpretation manual. $2^{\text {nd }}$ ed. Melbourne: CSIRO Publishing, 1997. 572p.

SACRAMENTO, C.K.; PEREIRA F.M. Fenologia da floração nogueira macadâmia (Macadamia integrifolia Maiden \& Betche) nas condições climáticas de Jaboticabal, São Paulo, Brasil. Revista Brasileira de Fruticultura, v.25, p.19-22, 2003. DOI: 10.1590/ S0100-29452003000100007.

SANTOS, H.G. dos; JACOMINE, P.K.T.; ANJOS, L.H.C. dos; OLIVEIRA, V.A. de; OLIVEIRA, J.B. de; COELHO, M.R.; LUMBRERAS, J.F.; CUNHA, T.J.F. (Ed.). Sistema brasileiro de classificação de solos. 2.ed. Rio de Janeiro: Embrapa Solos, 2006. $306 \mathrm{p}$.

SÃO PAULO (Estado). Secretaria de Agricultura e Abastecimento. Coordenadoria de Assistência Técnica Integral. Levantamento censitário de unidades de produção agrícola no Estado de São Paulo - LUPA 2007/2008. São Paulo: SAA/CATI/IEA, 2008. Disponível em: <http://www.cati.sp.gov.br/projetolupa/>. Acesso em: 10 abr. 2012.

SCHNEIDER, L.M.; ROLIM, G. de S.; SOBIERAJSKI, G. da R.; PRELA-PANTANO, A.; PERDONÁ, M.J. Zoneamento agroclimático de nogueira-macadâmia para o Brasil. Revista Brasileira de Fruticultura, v.34, p.515-524, 2012. DOI: 10.1590/ S0100-29452012000200025.

SOBIERAJSKI, G. da R.; FRANCISCO, V.L.F. dos S.; FAGUNDES, P.R.S.; GHILARDI, A.A.; MAIA, M.L. Noz-macadâmia: produção, mercado e situação no Estado de São Paulo. Informações Econômicas, v.36, p.25-36, 2006.

STEPHENSON, R.A.; CULL, B.W. Standard leaf nutrient levels for bearing macadamia trees in south east Queensland. Scientia Horticulturae, v.30, p.73-82, 1986. DOI: 10.1016/0304-4238(86)90083-X.

STEPHENSON, R.A.; GALLAGHER, E.C. Timing of nitrogen application to macadamias. 1. Tree nitrogen status and vegetative growth. Australian Journal of Experimental Agriculture, v.29, p.569-585, 1989. DOI: 10.1071/EA9890569.

STEPHENSON, R.A.; GALLAGHER, E.C.; DOOGAN, V.J. Leaf nitrogen as a guide for fertilizing macadamia. Australian Journal of Experimental Agriculture, v.37, p.599-604, 1997. DOI: 10.1071/EA96143. 
STEPHENSON, R.A.; GALLAGHER, E.C.; GOGEL, B.J. Macadamia nut size and maturity influenced by lime and nitrogen applications. Australian Journal of Agricultural Research, v.53, p.677-680, 2002. DOI: 10.1071/AR01146.
TROCHOULIAS, T.; JOHNS, G.G. Poor response of macadamia (Macadamia integrifolia Maiden and Betche) to irrigation in a high rainfall area of subtropical Australia. Australian Journal of Experimental Agriculture, v.32, p.507-512, 1992. DOI: 10.1071/EA9920507.

Recebido em 29 de novembro de 2012 e aprovado em 21 de março de 2013 